\title{
Overexpression of Candida albicans secretory aspartyl proteinase 2 and its expression in Saccharomyces cerevisiae do not augment virulence in mice
}

\author{
Nathalie Dubois, Ana Rosa Colina, Francine Aumont, Pierre Belhumeur \\ and Louis de Repentigny
}

Author for correspondence: Louis de Repentigny. Tel : +1 5143454643 . Fax: +1 5143454860 .
e-mail: louisr@globale.net

Department of

Microbiology and

Immunology, Faculty of

Medicine, University of

Montreal and Sainte-

Justine Hospital, 3175 Cóte

Sainte-Catherine,

Montreal, Québec

H3T 1C5, Canada

\begin{abstract}
To elucidate the implications of secreted aspartyl proteinase (Sap)2p in the pathogenesis of Candida infections, the SAP2 gene was expressed in Saccharomyces cerevisiae and overexpressed in Candida albicans. The coding region of SAP2, including its signal sequence and propeptide, was amplified by PCR and cloned downstream of the S. cerevisiae or C. albicans ADH1 promoter. Plasmid expression of SAP2 in S. cerevisiae showed that the signal peptide was functional. Integrative transformation of $S$. cerevisiae and $C$. albicans was accomplished by homologous recombination within the URA3 locus for $S$. cerevisiae and the SAP2 locus for C. albicans. Negative control transformants carried plasmids either without the SAP2 insert or with mutated sap2. S. cerevisiae and $C$. albicans transformants showed similar growth rates to their parental strains or negative controls, when grown in medium containing amino acids. However, in medium with BSA as sole nitrogen source, constitutive expression of SAP2 enabled S. cerevisiae to grow and increased the growth rate of $\mathrm{C}$. albicans. In both media, only 5 . cerevisiae transformants harbouring SAP2 secreted the enzyme, as confirmed by proteinase activity assays and immunoblotting. When $C$. albicans was grown in amino acids medium, the enzyme was detected exclusively in transformants constitutively expressing SAP2. However, in BSA medium these strains secreted enzyme earlier and secreted higher amounts of enzyme and total proteinase activity. In pathogenicity studies in intact mice, expression of Sap2p as a sole putative virulence factor did not cause $S$. cerevisiae to become virulent and constitutive overexpression of SAP2 did not augment virulence of $C$. albicans in experimental oral or systemic infection.
\end{abstract}

Keywords: candidiasis, Candida albicans, aspartyl proteinase, Saccharomyces cerevisiae

\section{INTRODUCTION}

Candida albicans is an important opportunistic pathogen causing local or systemic infection in immunocompromised patients (Odds, 1988). Several virulence factors have been proposed in pathogenicity: adhesion, dimorphism, phenotypic switching, molecular mimicry of mammalian integrins and secretion of phospholipases

Abbreviation: YNB, Yeast Nitrogen Base. and aspartyl proteinases (Cutler, 1991; White et al., 1995). The secreted aspartyl proteinases (Saps) have been studied extensively and are encoded by at least eight genes which are expressed and regulated differentially (Hube, 1996). Northern analysis has shown that $S A P 1$ and SAP3 are regulated during phenotypic switching between the white and opaque forms of strain WO-1, while SAP2 was found to be the dominant transcript in budding cells grown in medium containing protein as sole nitrogen source. The expression of SAP4-6 is detected at neutral $\mathrm{pH}$ during serum-induced 
yeast to hyphal transition, but the expression of SAP7 has not been detected (Hube et al., 1994; White \& Agabian, 1995).

In clinical and laboratory strains grown as the yeast form in vitro, Sap $2 p$ is the predominantly expressed proteinase. Evidence has also been provided that Saps are secreted by C. albicans in vivo during the course of mucosal and deep-tissue infections (Borg \& Rüchel, 1988; De Bernardis et al., 1995). The most commonly used approach to define the role of Saps as virulence attributes has been to compare the lethal effect on mice infected intravenously with parental strains or proteinase-deficient mutants. The mutants, isolated by chemical mutagenesis techniques or UV-irradiation, have been shown to be less virulent than their parental strains. However, definitive proof of the role of Saps in virulence of $C$. albicans has been lacking, because either (i) the mutants had high levels of reversion, (ii) they still produced detectable proteinase activity in vitro, (iii) the nature of the mutation was undefined or (iv) they may harbour multiple, independent and unknown lesions (Macdonald \& Odds, 1983; Kwon-Chung et al., 1985; Crandall \& Edwards, 1987; Ross et al., 1990).

Recently, C. albicans strains harbouring targeted disruptions of $S A P$ genes have been constructed using the ura-blaster protocol (Hube et al., 1997; Sanglard et al., 1997). When tested in a murine model of systemic candidiasis, the virulence of C. albicans sap1, sap2 and sap3 mutants was modestly attenuated (Hube et al., 1997). However, the extent of attenuation of virulence did not correlate directly with the reduction of proteolytic activity in vitro. Similarly, mice infected with a C. albicans sap4,5,6 triple homozygous null mutant had survival times significantly longer than that of control animals (Sanglard et al., 1997). In Candida tropicalis, disruption of the SAPT gene was also achieved by cotransformation with a linear DNA fragment carrying a deletion in SAPT (Sanglard et al., 1992). Surprisingly, the virulence of the sapT-null mutant and the proteinase-positive strain did not differ significantly after intravenous infection in mice (Togni et al., 1994). These results suggested that the Sap of C. tropicalis does not contribute significantly to fungal virulence in systemic infections. Alternatively, potentially decreased virulence may have been masked by the redundancy of members of a putative multigene Sap family in C. tropicalis (Monod et al., 1994) and/or the redundancy of other virulence attributes of Candida.

Selective expression/overexpression of genes encoding putative virulence attributes is an attractive strategy to overcome redundancy problems and clarify the contribution of each isoenzyme to virulence. This approach would allow the study of virulence-enhancing genes in already virulent strains (homologous overexpression) and in non-pathogenic but closely related strains (heterologous expression). The rationale for this approach is also based on clinical studies which have shown that the Sap activity of C. albicans isolates from patients with vaginitis (Cassone et al., 1987) or HIV infection (Ollert et al., 1995) was significantly higher than that of isolates from asymptomatic carriers. In this study, we describe the construction of stable Saccharomyces cerevisiae and C. albicans strains that constitutively secrete Sap2p. Sap2p expression by $S$. cerevisiae allowed it to hydrolyse and use BSA as sole nitrogen source, while Sap2p overexpression in C. albicans resulted in early and highlevel proteinase secretion. The virulence of these genetically engineered strains was evaluated in murine models of oral or systemic infections.

\section{METHODS}

Strains and culture conditions. Yeast strains used in this work are listed in Table 1. Escherichia coli DH10B (Gibco-BRL) was routinely used as a plasmid host for cloning procedures. Bacterial and yeast media were prepared as described by Rose et al. (1990) and Sambrook et al. (1989). Yeast parental strains were grown in YPD medium containing $1 \%(\mathrm{w} / \mathrm{v})$ yeast extract (Difco), $2 \%(\mathrm{w} / \mathrm{v}$ ) Bacto peptone (Difco) and $2 \%$ $(\mathrm{w} / \mathrm{v})$ dextrose. Uracil/uridine independence selection was performed by using CAT medium $[0.67 \%$ Yeast Nitrogen Base (YNB) without amino acids (Difco), 0.5 or $1 \%(\mathrm{w} / \mathrm{v}$ ) Casamino acids, $0.008 \%$ adenine, $0.002 \%$ tryptophan] supplemented with $2 \%$ dextrose. The medium to regenerate $C$. albicans spheroplasts was supplemented with $1 \mathrm{M}$ sorbitol. Induction of Sap2p was done in YNB-BSA medium containing $0 \cdot 17 \%$ YNB without amino acids and ammonium sulfate (Difco), supplemented with $2 \%$ dextrose and $0.2 \%$ BSA (fraction V; Sigma). Media were supplemented with $100 \mu \mathrm{g}$ uracil/uridine $\mathrm{ml}^{-1}$ as required. All yeast cultures were incubated at $30^{\circ} \mathrm{C}$, except for experiments on the kinetics of Sap $2 p$ secretion by $S$. cerevisiae and C. albicans which were conducted at $37^{\circ} \mathrm{C}$. Growth rates were monitored by measuring $\mathrm{OD}_{600}$. Media were solidified with $2 \%(\mathrm{w} / \mathrm{v})$ agar. Germ tube formation was evaluated by incubating in $5 \%$ (v/v) newborn calf serum (Gibco-BRL) for $3 \mathrm{~h}$ at $37^{\circ} \mathrm{C}$.

PCR amplification of SAP2 gene. Genomic DNA from C. albicans LAM-1, obtained as described by Magee et al. (1987), was used as template for PCR amplification of $S A P 2$. The two primers used were $5^{\prime}$ CGGGATATCAACAACCCACTAGACATCACC and 5' CTGGAGCTCCACCCCTTCATCTTAGGTCAA. These primers flank the SAP2 coding sequence with its own signal peptide. The underlined sequences indicate $E c o R V$ and SacI restriction sites, respectively. The $100 \mu \mathrm{l} \mathrm{PCR}$ reaction contained $1 \mu \mathrm{g}$ genomic DNA template, $1 \mu \mathrm{g}$ each of the two primers, $400 \mu \mathrm{M}$ each of dATP, dGTP, dCTP and dTTP, $10 \mu \mathrm{l}$ DMSO, $2 \mathrm{U}$ Vent DNA polymerase (New England Biolabs) and $1 \times$ Vent buffer. The PCR program was $94{ }^{\circ} \mathrm{C}$ for $1 \mathrm{~min}, 60^{\circ} \mathrm{C}$ for $1 \mathrm{~min}$ and $72{ }^{\circ} \mathrm{C}$ for $2 \mathrm{~min}$ for 30 cycles. A 1244 bp PCR product was gel-purified and cloned into pBluescript KS II (+) (Stratagene) after digestion with EcoRV and SacI. The PCR product sequence was verified by double-strand sequencing with universal and internal primers (Sanger et al., 1977).

Plasmid constructions. Recombinant DNA manipulations were done by standard methods (Sambrook et al., 1989). Plasmid pVTU-SAP2 was constructed by inserting the SAP2 PCR product, digested by EcoRV and Ecl136II, into the Ecl136II site of $S$. cerevisiae shuttle vector pVT102-U (Vernet et al., 1987). A negative control, pVTU-aSAP2, carried SAP2 in antisense orientation. pVT102-U is a $2 \mu$-based plasmid bearing the $A D H 1$ promoter $(A D H 1 \mathrm{p})$, its terminator region and the $U R A 3$ marker gene. From pVTU-SAP2, a $2 \mathrm{~kb} S p h \mathrm{I}$ fragment containing $S A P 2$ downstream of $A D H 1 \mathrm{p}$, was bluntended with T4 DNA polymerase and inserted into the SmaI

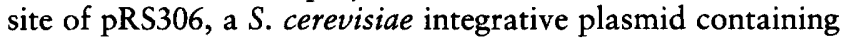


Table 1. Yeast strains

\begin{tabular}{|c|c|c|}
\hline Strain & Genotype & Source or reference \\
\hline \multicolumn{3}{|l|}{ S. cerevisiae } \\
\hline cIABYS86 & MAT leu2 ura3-52 bis3 pra1 prb2 prc1 cps & A. H. Bussey (McGill University, Canada) \\
\hline PUB754 & MAT $\alpha$ ura3-52 pra1 prb2 prc1 cps & This study \\
\hline SEY6210 & MAT $\alpha$ leu2-3,112 ura3-52 his $3 \Delta 200$ lys $2-801$ trp1 1901 suc2 $2 \Delta 9$ & Robinson et al. (1988) \\
\hline Y55 & Laboratory isolate, $\mathrm{HO}$ & Clemons et al. (1994) \\
\hline YJM128 & Clinical isolate & Clemons et al. (1994) \\
\hline YJM273 & Clinical isolate & Clemons et al. (1994) \\
\hline \multicolumn{3}{|l|}{ C. albicans } \\
\hline CAI4 & $\Delta u r a 3:: i m m 434 / \Delta u r a 3:: i m m 434$ & Fonzi \& Irwin (1993) \\
\hline LAM-1 & Clinical isolate & Lacasse et al. (1990) \\
\hline SC5314 & Clinical isolate & Gillum et al. (1984) \\
\hline
\end{tabular}

the URA3 marker gene (Sikorski \& Hieter, 1989). This plasmid was named pRS-SAP2. Finally, the C. albicans vector containing C. albicans $A D H 1 \mathrm{p}$ and $U R A 3$ marker, YPB-ADHpL (kindly provided by A. J. P. Brown, University of Aberdeen, UK), was used to insert the 1244 bp EcoRV-Ecl136II SAP2 fragment into EcoRV site under the control of $C$. albicans $A D H 1 \mathrm{p}$. This construct was named pYPB-SAP2. A negative control plasmid, pYPB-mSAP2, was generated by inserting a mutated gene, sap2. The mutation was introduced by cleavage of SAP2 with BamHI, filled-in with Klenow DNA polymerase and religated. This introduced a frameshift mutation (verified by sequencing) after the sequence encoding aa 77 and a premature stop codon 9 bp ( $3 \mathrm{aa}$ ) further downstream.

Yeast transformation. $S$. cerevisiae strains grown overnight in YPD medium at $30^{\circ} \mathrm{C}$, were transformed using the lithium acetate/ssDNA/PEG procedure described by Schiestl et al. (1993). Strains cIABYS86 (S86), PUB754 (754) and SEY6210 (6210) were transformed with pVTU-SAP2. PUB754, a His ${ }^{+}$ $\mathrm{Leu}^{+}$derivative of S86, was obtained by two separate 'onestep gene replacement' transformations with HIS3 and LEU2 DNA fragments (Rothstein, 1983). Integration of SAP2 in the S. cerevisiae URA3 locus was done by transforming strain 754 with pRS-SAP2, linearized by $\mathrm{NcoI}$ in URA3. Negative controls were also constructed by transforming strain 754 with NcoI-linearized plasmid pRS306.

Transformation of C. albicans CAI4 was done by the spheroplast method as described by Rose et al. (1990), with minor modifications. Briefly, CAI4 yeast cells grown overnight in YPD were diluted in YNB-BSA medium (supplemented with uridine) to an $\mathrm{OD}_{600}$ of $0 \cdot 2$. Then, exponentially growing cells $\left(\mathrm{OD}_{600}\right.$ of 0.5$)$ were used to prepare the spheroplasts. pYPB-SAP2 and pYPB-mSAP2 plasmids were linearized by cutting at the KpnI site within $S A P 2$ and used to transform the spheroplasts by integration at the $S A P 2$ locus. The $S$. cerevisiae and $C$. albicans transformants, selected by complementation of the ura3 mutation, were grown on CAT plates.

Proper integration was evaluated by Southern blotting analysis (Belhumeur et al., 1993). Genomic DNA from S. cerevisiae transformants, prepared as described by Hoffman \& Winston (1987), was digested with AvrII and probed with a 693 bp EcoRV-SmaI URA3 (see Fig. 2a) or a 597 bp BamHI-KpnI $S A P 2$ restriction fragment. C. albicans genomic DNA, prepared according to Magee et al. (1987), was digested with KpnI and probed with a 265 bp PstI-DraI SAP2 DNA fragment (see Fig. 2b).

SDSPAGE and Western blotting. Intra- and extracellular protein extracts from $S$. cerevisiae plasmid transformants grown for $18 \mathrm{~h}$ in CAT medium at $30^{\circ} \mathrm{C}$ were obtained by lysing with glass beads (Harlow \& Lane, 1988) and acetone precipitation, respectively. One-tenth of the original extract volume was loaded in a $12 \%$ polyacrylamide separating gel to perform SDS-PAGE (Laemmli, 1970). For time course evaluation of Sap2p expression, $60 \mu$ l culture supernatants was analysed by SDS-PAGE. Following electrophoresis, proteins were transferred onto nitrocellulose membranes (Towbin et al., 1979). Membranes were blocked with $2 \%$ (w/v) skim milk in Tris-buffered saline (TBS: $10 \mathrm{mM}$ Tris/ $\mathrm{HCl}, \mathrm{pH} 7 \cdot 5$, $150 \mathrm{mM} \mathrm{NaCl}$ ) and washed between steps with TBS containing $0.1 \%$ Tween 20 (TBST). Membranes were incubated for $1 \mathrm{~h}$ with mouse anti-Sap2p mAb IFG3, kindly provided by T. L. Ray (University of Iowa), diluted $1: 500$ in TBS $/ 2 \%$ skim milk. The bound antibody was detected using alkalinephosphatase-conjugated goat anti-mouse IgG (Gibco-BRL) diluted $1: 5000$ in TBS $/ 2 \%$ skim milk.

Enzyme assays. Proteolytic activity was quantified by the method of Milewski et al. (1994) with minor modifications. Briefly, culture supernatants $(150 \mu \mathrm{l})$ were mixed with $1 \%$ BSA in $50 \mathrm{mM}$ sodium citrate, pH $3.5(600 \mu \mathrm{l})$. After $1 \mathrm{~h}$ incubation at $37^{\circ} \mathrm{C}$, reactions were stopped by adding $400 \mu \mathrm{l}$ $10 \%(\mathrm{w} / \mathrm{v})$ TCA. Samples were cooled in an ice bath for $10 \mathrm{~min}$ and precipitated proteins were removed by centrifugation at $5000 \mathrm{~g}$ for $10 \mathrm{~min}$. The $A_{280}$ of the supernatants was measured and corrected for background using a control (for each point) in which TCA solution was added prior to the culture supernatant. One unit of enzyme activity was defined as the amount of enzyme causing a $\Delta A_{280}$ of $0 \cdot 1$ in $1 \mathrm{~h}$.

Pathogenicity in vivo. The virulence of $S$. cerevisiae and $C$. albicans transformants as well as their parental strains was evaluated in murine models of oral and systemic candidiasis. $\mathrm{Crl}$ :CD-1 (ICR) BR female mice, weighing 22-24 g, were obtained from Charles River Breeding Farms (St Constant, Québec, Canada) and were kept at the University of Montreal Animal Care Unit. For experimental infections, the yeast inoculum was prepared from cells grown overnight in CAT medium at $30^{\circ} \mathrm{C}$.

The oral infection model described by Chakir et al. (1994) was used with minor modifications. Briefly, both $S$. cerevisiae and C. albicans yeast cells were washed twice in sterile $0.01 \mathrm{M}$ PBS $(\mathrm{pH} 7 \cdot 4)$ and counted in a haemocytometer. Cells $\left(1 \times 10^{8}\right)$ were distributed in sterile plastic microfuge tubes and pelleted by centrifugation at $13000 \mathrm{~g}$. Six mice per strain were anaesthetized intraperitoneally with $0.35 \mathrm{ml}$ ketamine $\left(10 \mathrm{mg} \mathrm{ml}^{-1}\right)$. They were then inoculated by topical application into the oral cavity using a sterile calcium alginate swab 
(Fisher Scientific). A longitudinal quantification of the microorganisms in the oral cavities of individual mice was done by daily oral swabbing until $8 \mathrm{~d}$ post-inoculation. Swabs used for sampling were dissolved in $2 \mathrm{ml}$ volumes of Ringer's citrate buffer (Rodrigue et al., 1989) and serial dilutions in PBS were plated on CAT medium supplemented with $50 \mathrm{mg}$ chloramphenicol $1^{-1}$ (CAT-Chl). Plates were incubated at $30^{\circ} \mathrm{C}$ and c.f.u. were counted.

Experimental murine systemic infection (Clemons et al., 1994) was used to study $S$. cerevisiae virulence. Mice were inoculated intravenously with $2 \times 10^{7}$ or $6 \times 10^{7}$ cells, previously washed twice in sterile saline. Six mice per yeast strain were killed at 1,2 or 4 weeks after infection. To determine organ burden, brains and kidneys were removed aseptically, homogenized in $5 \mathrm{ml}$ sterile saline and plated on CAT-Chl and Sabouraud supplemented with chloramphenicol (SDA-CHL) for determination of c.f.u.

The virulence of $C$. albicans strains was also evaluated in a model of systemic candidiasis. Mice were inoculated intravenously with $5 \times 10^{5}$ cells, previously washed twice in PBS. Mice were observed daily for signs of morbidity over $15 \mathrm{~d}$. Moribund animals were scored as non-survivors and euthanized by $\mathrm{CO}_{2}$ inhalation. To evaluate tissue invasion, additional groups of infected mice were sacrificed at 1,3 or $6 \mathrm{~d}$ post-inoculation. One kidney from each animal was removed and prepared for histological analysis. They were fixed in $10 \%$ formalin, embedded in paraffin, sectioned and stained by the Grocott procedure (Luna, 1992). The remaining kidney and the liver were also removed aseptically, weighed and homogenized in $5 \mathrm{ml}$ PBS. Homogenates were plated on CAT$\mathrm{Chl}$ and SDA-Chl and incubated at $30^{\circ} \mathrm{C}$ for colony counting. Data were expressed as c.f.u. (g kidney or liver) ${ }^{-1}$. Several colonies of C. albicans were isolated from both media to verify the in vivo stability of SAP2 integration by Southern blotting as described above.

Zymolyase and $\mathrm{NaCl}$ resistance. A zymolyase test was used to compare the sensitivity of the cell wall of transformants and parental strains (Lussier et al., 1997). S. cerevisiae and C. albicans strains were grown in CAT medium to exponential phase $\left(\mathrm{OD}_{600}\right.$ of 0.6$)$, washed twice in sterile saline and resuspended in sterile zymolyase buffer $(50 \mathrm{mM}$ Tris $/ \mathrm{HCl}$, $\mathrm{pH} 7 \cdot 4)$ at an $\mathrm{OD}_{600}$ of 0.635 . Aliquots of $400 \mu \mathrm{l}$ were mixed with $100 \mu \mathrm{l}$ zymolyase $100 \mathrm{~T}\left(1 \mathrm{mg} \mathrm{ml}^{-1}\right)$ or $100 \mu \mathrm{l}$ zymolyase buffer (controls). After $1 \mathrm{~h}$ incubation at $37^{\circ} \mathrm{C}$ with gentle shaking, $5 \mu$ l serial dilutions were spotted on CAT agar medium. C. albicans and S. cerevisiae strains were also tested for $\mathrm{NaCl}$ resistance. Briefly, cultures grown overnight in CAT medium were patched onto CAT plates with $0,0.2,0.6,1 \cdot 0$, $1 \cdot 4,1 \cdot 8,2 \cdot 2$ or $2 \cdot 6 \% \mathrm{NaCl}$. Sensitivity to zymolyase and $\mathrm{NaCl}$ was determined by comparison of the level of growth after incubation at $30^{\circ} \mathrm{C}$.

Statistical analysis. The data were fed to the SAS system (SAS Institute, Cary, NC, USA). The Kaplan \& Meier (1958) product limit estimate was used to analyse survival data and plot the survival function. The Wilcoxon method (DawsonSaunders \& Trapp, 1990) was applied to compare the survival functions of experimentally infected mouse populations and differences were considered significant at the 0.01 level.

Differences in weights and c.f.u. (g kidney) ${ }^{-1}$ were compared among mouse populations by using the general linear models procedure two-way analysis of variance, conducted with two factors, one between (strain) and one within (time). Significant interactions $(P<0.01)$ were further analysed using the Bonferroni (Dunn) $t$-test (Dawson-Saunders \& Trapp, 1990) and the resulting comparisons were considered significant at the 0.01 level.

\section{RESULTS}

\section{Expression and secretion of Sap2p by S. cerevisiae}

As an initial approach toward heterologous expression of C. albicans SAP2 in S. cerevisiae, we performed a PCR amplification of this gene from C. albicans LAM-1. The $1244 \mathrm{bp}$ PCR product containing the native prepro and mature coding regions was cloned into the multicopy expression vector $\mathrm{pVT} 102-\mathrm{U}$ downstream of the constitutive $A D H 1$ promoter, to create the plasmid pVTUSAP2 (sense orientation). A negative control, pVTUaSAP2 (antisense orientation), bearing the PCR product in opposite orientation was also constructed. These recombinant plasmids were transformed into $S$. cerevisiae 6210 and S86, the latter defective in intracellular proteinases (Table 1).

The expression of Sap2p was evaluated by Western blotting in intra- and extracellular extracts. As shown in Fig. 1 (lanes 3, 4 and 6), a single positive signal of approximately $42 \mathrm{kDa}$ was detected in the extracts of strains transformed with pVTU-SAP2 (sense orientation). A decreased loading revealed additional forms of 39 and $45 \mathrm{kDa}$ (data not shown). The largest amount of Sap2p was detected in the extracellular fraction (secreted), indicating that the secretion signal was fully functional in S. cerevisiae. In addition, there was more abundant production of Sap2p by strain S86, the intracellular proteinases mutant, compared to 6210 (Fig. 1 , lanes 4 and 6 ). We could not determine whether this difference was due to the lack of these proteinases, since these strains are not isogenic. In addition, degradation of casein was demonstrated in a Petri dish assay (Colina et al., 1996a) using unconcentrated culture supernatant

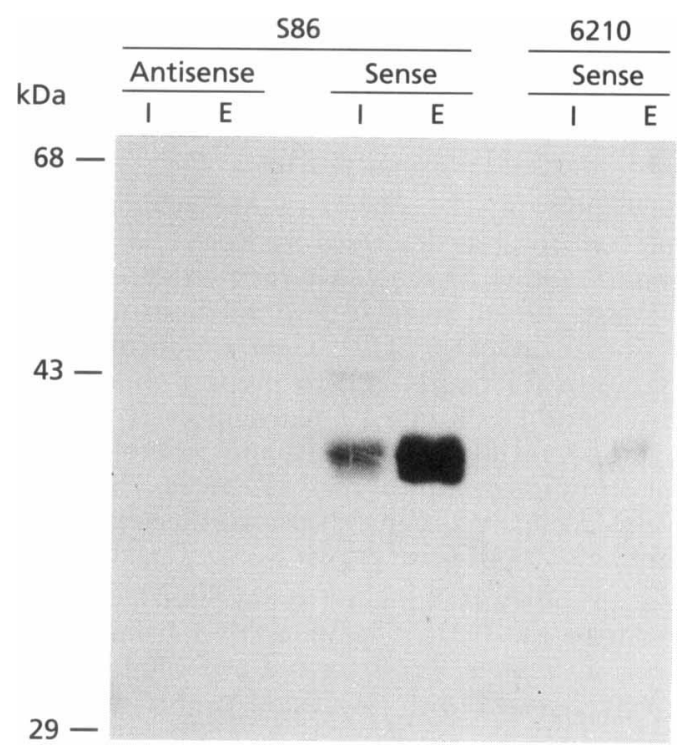

Fig. 1. Western blot analysis of intra- (I) and extracellular (E) proteins of S. cerevisiae $\mathbf{S 8 6}$ and 6210 plasmid transformants (with sense or antisense SAP2 constructs) grown in CAT medium for $18 \mathrm{~h}$ at $30^{\circ} \mathrm{C}$ and probed with an anti-Sap2p mAb. Loaded sample volumes were adjusted to equivalent mass cultures. 
(a)
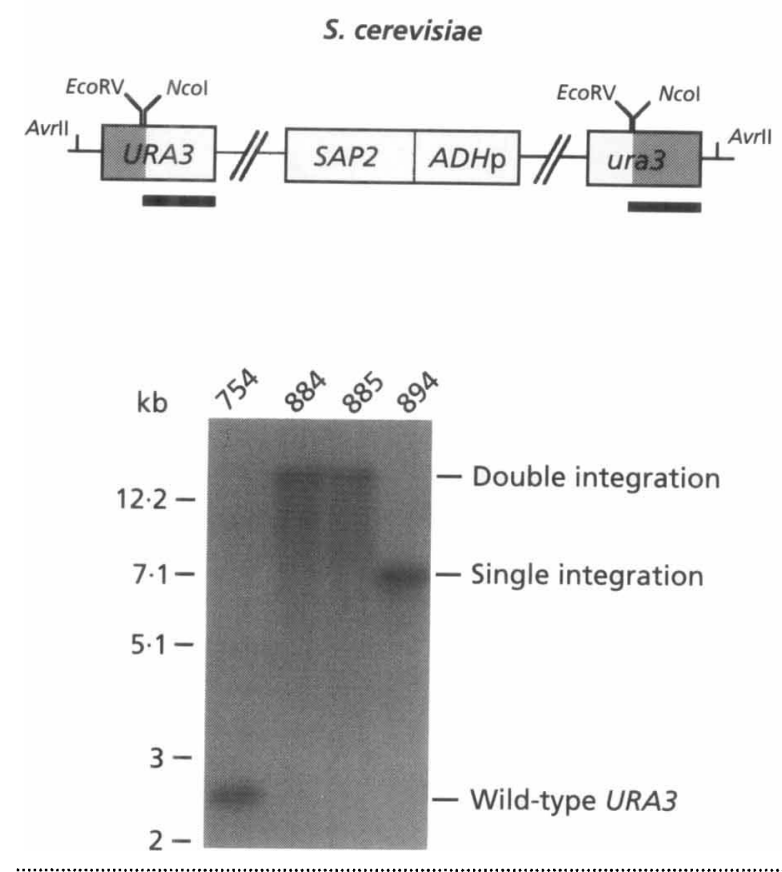

(b)
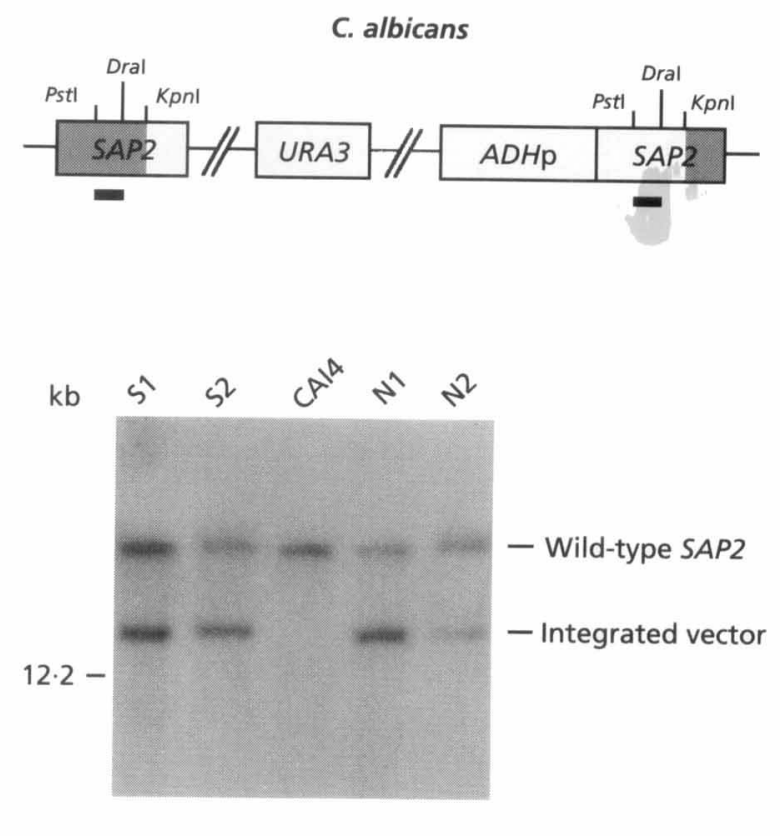

Fig. 2. Southern blot analysis of wild-type and selected transformants of (a) S. cerevisiae and (b) C. albicans. Top, integration sites with probes indicated by thick lines. Bottom, S. cerevisiae and C. albicans genomic DNAs digested with Avrll and Kpnl, respectively, and probed with ${ }^{32}$ P-labelled EcoRV-Smal (S. cerevisiae) or Pstl-Dral SAP2 (C. albicans) restriction fragments. Shaded areas represent the chromosomal copy of URA3 and SAP2.

of $S 86$ (sense orientation), but was absent in the antisense orientation (data not shown). Based on these results, we decided to select strain S86 harbouring pVTU-SAP2 for further experiments. No anti-Sap2p-reacting material was detected in the strain transformed with pVTUaSAP2 (antisense orientation; Fig. 1, lanes 1 and 2).

Overall, these findings indicated that the recombinant enzyme was efficiently expressed and secreted into the culture medium and that the signal sequence of $C$. albicans SAP2 was functional in S. cerevisiae.

\section{Integrative transformation of S. cerevisiae and C. albicans}

To produce stable $S$. cerevisiae and C. albicans transformants that could be used in animal models and for further genetic analysis, plasmids carrying SAP2 were integrated into the genome by homologous recombination.

(i) S. cerevisiae. The integrative plasmid, pRS-SAP2 (see Methods), was linearized at the NcoI site in URA3 and transformed into strain 754 . A negative control was obtained by integrating pRS306 without insert. Proper integration was evaluated by Southern blotting after digestion of parental and transformant genomic DNAs with AvrII. As shown in Fig. 2(a), a single $2.7 \mathrm{~kb}$ fragment hybridized to the URA3 probe in the parental strain (754) as predicted. In positive transformants (884,
885), we expected a $9 \cdot 1 \mathrm{~kb}$ fragment, resulting from the integration of pRS-SAP2. Unexpectedly, both strains gave a positive signal larger than $15 \mathrm{~kb}$ which can be interpreted as a double integration. Because of satisfactory expression of Sap $2 p$, these strains were selected for further studies (see below). A $7 \cdot 1 \mathrm{~kb}$ band was detected in negative control 894, corresponding to the integrated vector without SAP2. These findings were confirmed by probing the same blot with a radiolabelled SAP2 DNA fragment (data not shown).

(ii) C. albicans. Plasmids pYPB-SAP2 and pYPB-mSAP2 carrying the wild-type and mutated $S A P 2$, respectively, under the control of $A D H 1 \mathrm{p}$ were linearized at the $K p n \mathrm{I}$ site to target the integration into the SAP2 locus. Genomic DNAs were digested with $K p n I$ and probed with the SAP2 fragment (see Methods). In the parental strain (CAI4), the SAP2 probe hybridization resulted in a single band larger than $13 \mathrm{~kb}$ derived from the wildtype alleles of SAP2. The transformants $\mathrm{S} 1, \mathrm{~S} 2, \mathrm{~N} 1$ and $\mathrm{N} 2$ had an additional $13 \mathrm{~kb}$ fragment, which corresponds in size to the integrated construct (Fig. 2b).

\section{Kinetics of Sap2p secretion by S. cerevisiae}

The time course for proteinase secretion was investigated in media containing free amino acids from acid hydrolysis of casein (CAT), or BSA as nitrogen source (YNB-BSA). In CAT medium, positive transformants 884 and 885 and negative control 894 showed growth 
(a)

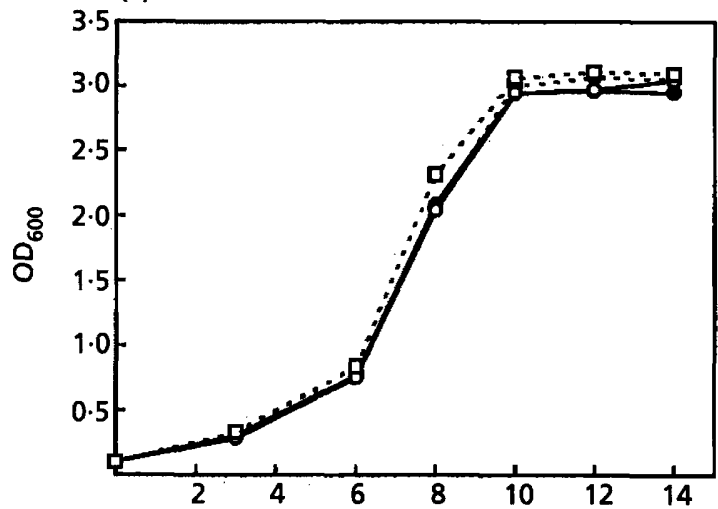

(b)

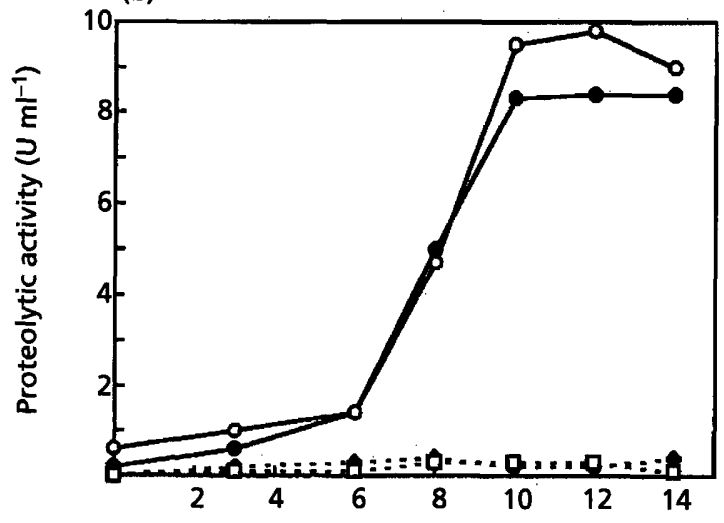

YNB-BSA
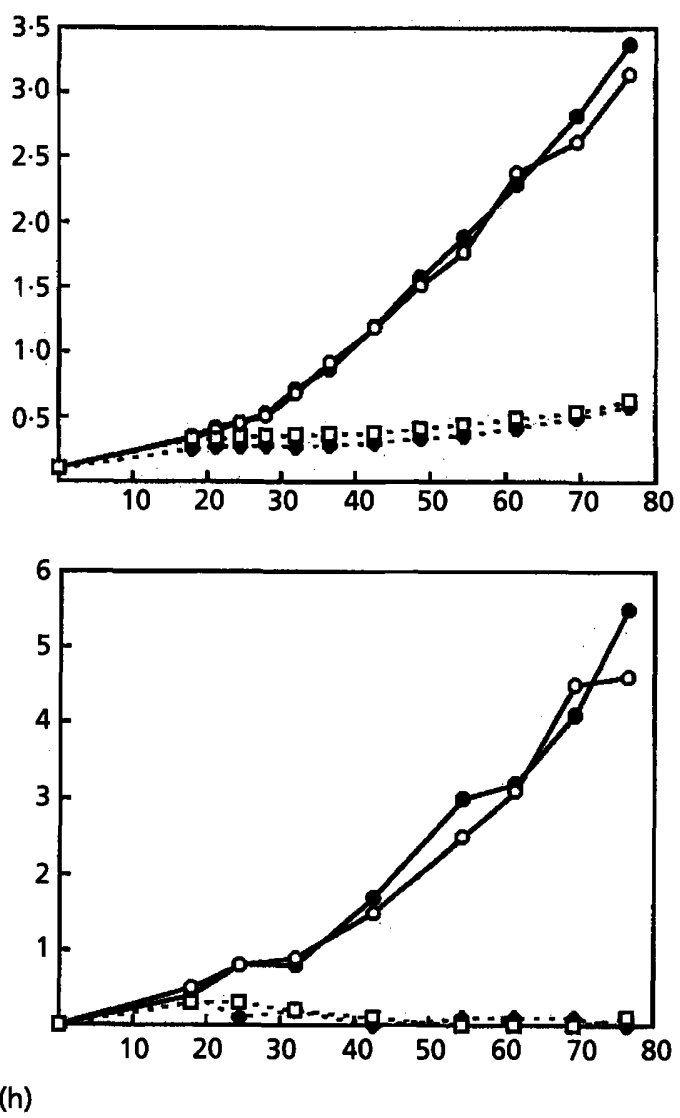

(c)

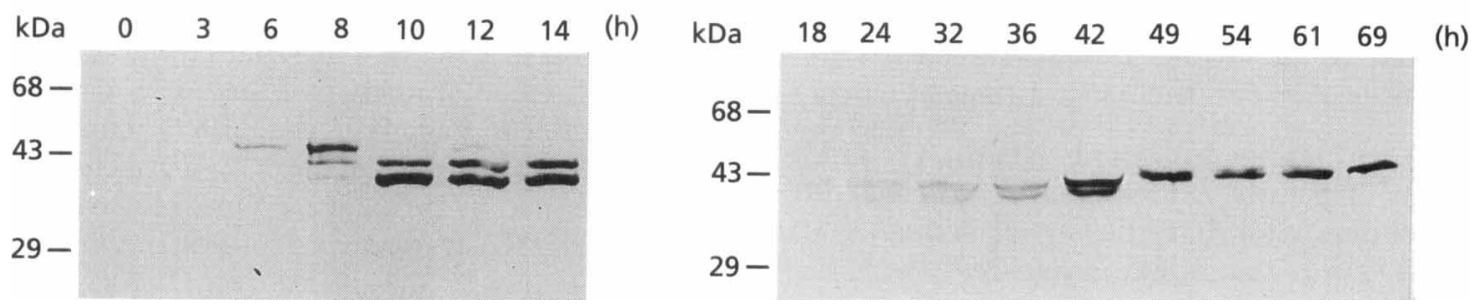

Fig. 3. Kinetics of Sap2p secretion by S. cerevisiae positive transformants $884(0)$ and $885(O)$, negative control 894 ( $\square$ ) and parental strain $754(\diamond)$ grown in $0.5 \%$ CAT (left) or YNB-BSA (right) media at $37^{\circ} \mathrm{C}$. At the indicated times, culture samples were evaluated for growth (a), extracellular proteolytic activity (b) and Sap2p expression in 885 (Western blot) (c). Data are mean values of duplicate experiments.

rates similar to parental strain 754 (Fig. 3a, left). Growth in YNB-BSA medium was restricted to strains harbouring $S A P 2$, suggesting their ability to use BSA as sole nitrogen source (Fig. 3a, right). However, it is noteworthy to mention that the growth rates in this medium were lower than in CAT.

In both media, secreted proteolytic activity was detected exclusively in those transformants carrying SAP2 (Fig. $3 b$ ). However, the level of activity was $1 \cdot 7$-fold higher in CAT than in YNB-BSA for the same cell density. This could reflect the greater physiological activity of the cells grown in the former medium. In CAT medium, the maximum level of proteinase activity was attained at the beginning of the stationary phase (Fig. 3b, left).

Additional information on Sap2p secretion was obtained from Western blotting analysis of 885 transformant supernatants. Sap2p was detectable at the beginning of the exponential phase, after 6 and $24 \mathrm{~h}$ of incubation in CAT and YNB-BSA media, respectively (Fig. 3c). In both media, the protein was processed to three forms 
(a)

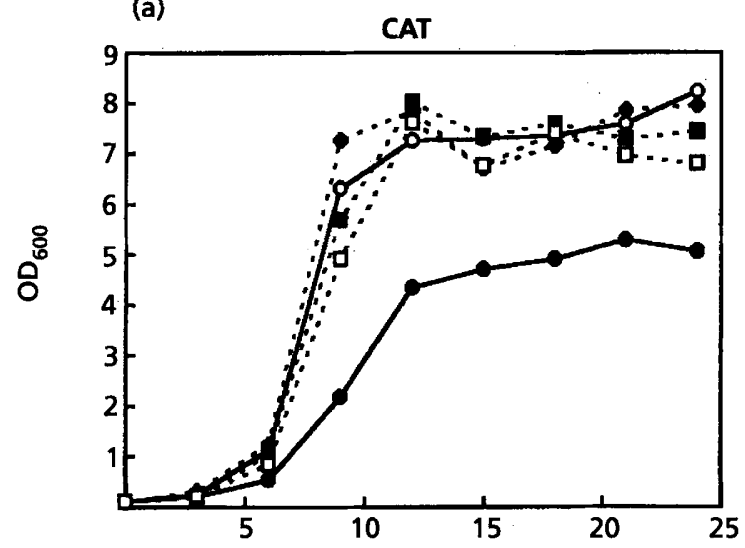

(b)

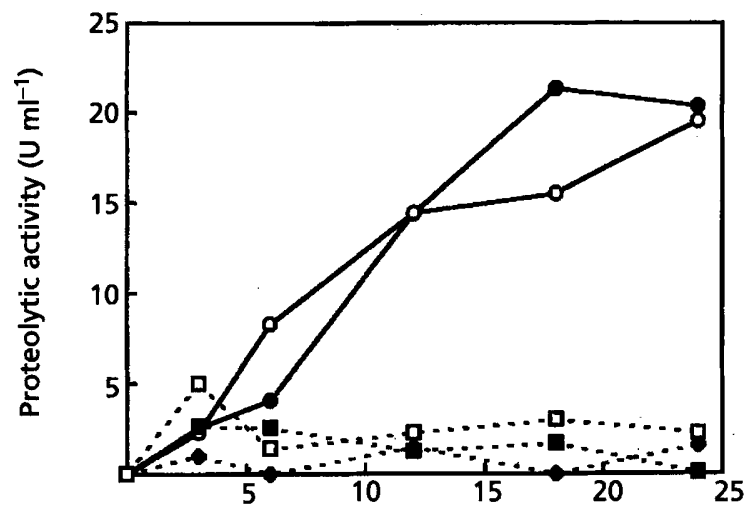

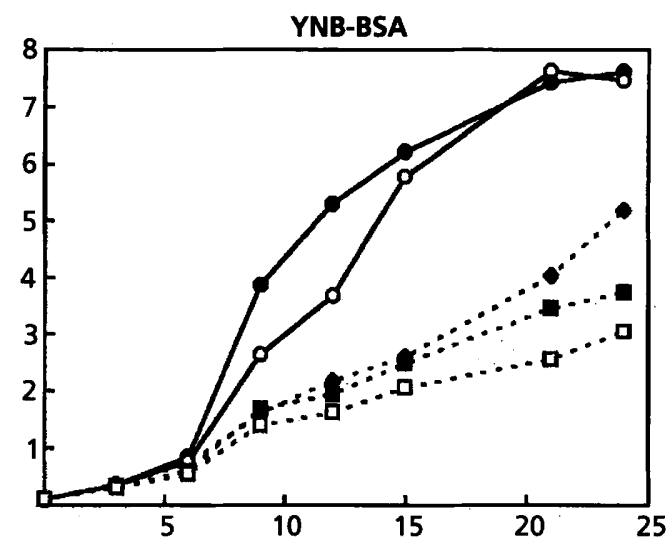

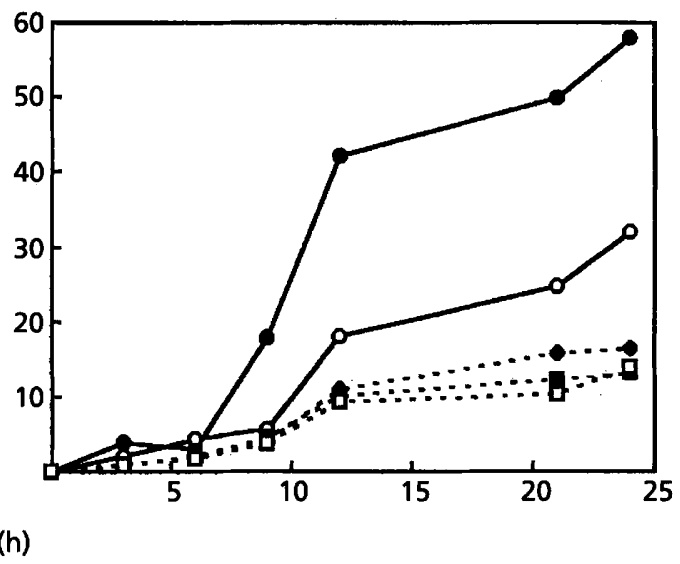

(c)
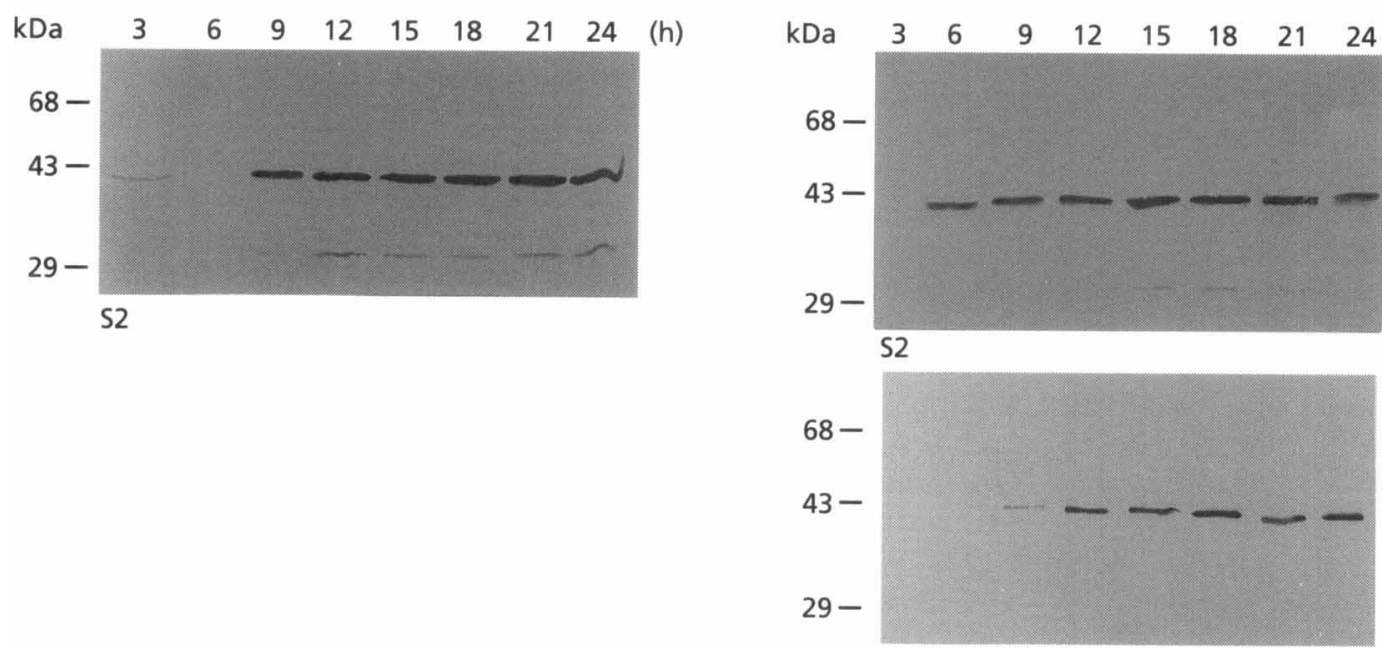

(h)

N1

Fig. 4. Kinetics of Sap2p secretion by $C$. albicans positive transformants $S 1(O)$ and $S 2(O)$, negative controls $N 1$ ( $\square$ ) and N2 $(\square)$, and parental strain CAI4 $(\bullet)$ grown in $1 \%$ CAT (left) or YNB-BSA (right) media at $37^{\circ} \mathrm{C}$. At the indicated times, culture samples were evaluated for growth (a), extracellular proteolytic activity (b) and Sap2p expression in S2 and N1 (Western blot) (c). Data are mean values of duplicate experiments. 
with approximate molecular masses of 45,42 and $39 \mathrm{kDa}$. The middle $42 \mathrm{kDa}$ form co-migrated with Sap2p purified from concentrated culture filtrate of $C$. albicans LAM-1, consistent with our previous observations (Colina et al., 1996b). The larger $45 \mathrm{kDa}$ form may result from altered post-translational modification or incomplete processing of the protein by $S$. cerevisiae and the smaller $39 \mathrm{kDa}$ form may represent a degradation product. Using a zymogram procedure described previously (Colina et al., 1996b), it was shown that each of the three bands had enzymic activity using mucin as substrate (data not shown). No Sap2p was detected in negative control 894 (data not shown).

\section{Kinetics of Sap2p secretion by C. albicans}

As for $S$. cerevisiae, C. albicans was grown in CAT or YNB-BSA medium, not inducing or inducing SAP2 expression, respectively. In CAT medium, the growth rates of the secretor-transformant $\mathrm{S} 2$, as well as the negative controls $\mathrm{N} 1$ and $\mathrm{N} 2$ (with mutated sap2), were indistinguishable from that of the parental strain, CAI4. However, the secretor-transformant S1 grew far more slowly than CAI4 (Fig. 4a, left). In YNB-BSA medium, transformants $\mathrm{S} 1$ and $\mathrm{S} 2$ displayed similar growth rates, which were greater than those of CAI4 and negative controls (Fig. 4a, right). Overall, cultures of S2, N1, N2 and CAI4 reached a higher cell density when media were supplemented with amino acids rather than BSA as nitrogen source.

Under non-inducing conditions (CAT medium), secreted proteolytic activity was detectable only in the supernatants of transformants S1 and S2 (Fig. 4b, left). These results indicated that $S A P 2$, placed under $A D H 1 \mathrm{p}$, was constitutively expressed in transformants $\mathrm{S} 1$ and $\mathrm{S} 2$. In YNB-BSA medium, conditions in which wild-type SAP2 is induced, proteolytic activity was detected in CAI4 and negative controls as well as in secretortransformants. However, in the latter strains, activity was $3 \cdot 3$-fold (S1) and 1.75-fold (S2) higher than in CAI4 or negative controls (Fig. 4b, right). Although higher proteolytic activity in S1 could have possibly resulted from the integration of multiple copies of the $A D H 1$ fusion, this was unlikely because the intensities of the bands for wild-type $S A P 2$ and integrated vector were individually comparable in the Southern analysis of S1 and S2 (Fig. 2b). Enhanced proteolytic activity in YNBBSA compared to CAT medium (Fig. 4) may have resulted from the presence of other Saps produced in YNB-BSA (Hube et al., 1994).

Finally, the supernatants of S2 and N1 strains were selected to evaluate the presence of Sap2p. In CAT medium, Sap2p of S2 (apparent molecular mass $42 \mathrm{kDa}$ ) was detectable after $9 \mathrm{~h}$ incubation, corresponding to the exponential phase (Fig. 4c, left). No protein was detected in N1 supernatants (data not shown). Interestingly, when BSA was included in the culture medium (Fig. 4c, right), S2 produced detectable Sap2p $3 \mathrm{~h}$ earlier than N1. The slower growth rate of N1 compared to S2 correlated with the apparent lower level of secretion of Sap2p.

Germination rates of the transformants and parental strain were indistinguishable. After $3 \mathrm{~h}$ of incubation at $37^{\circ} \mathrm{C}$, more than $70 \%$ of blastoconidia had germinated.

\section{Pathogenicity in vivo}

The virulence of $S$. cerevisiae negative (894) and positive $(884,885)$ transformants was evaluated in experimental oral or systemic infection in mice. In the former, all three strains were completely cleared from the oral cavity $5 \mathrm{~h}$ after inoculation (data not shown). A similar result was obtained after systemic infection. No c.f.u. were recovered from kidneys or brains of mice over a period of $28 \mathrm{~d}$ after infection with each of the $S$. cerevisiae strains. The inoculum of $2 \times 10^{7}$ cells was selected in accordance with a study by Clemons et al. (1994) which evaluated the pathogenic potential of various clinical and laboratory isolates of $S$. cerevisiae. In addition, when the experiment was repeated using $6 \times 10^{7}$ cells to exclude the possibility of an insufficient inoculum, cultures of homogenized organs remained completely negative for all strains. A control experiment was conducted using a $2 \times 10^{7}$ cells inoculum of $S$. cerevisiae Y55 (avirulent laboratory strain) and virulent clinical isolates YJM128 and YJM273, as described by Clemons et al. (1994). Quantification of c.f.u. in kidneys and brains of mice produced results very close to those reported by Clemons et al. (1994) (data not shown). This confirmed that lack of virulence with strains 884,885 and 894 was not due to a technical problem.

C. albicans parental strain SC5314 and positive (S1, S2) and negative (N1, N2) transformants were compared for their virulence in two models of candidiasis. In the murine oral model, all four transformants were completely cleared $24 \mathrm{~h}$ post-inoculation. Positive control C. albicans LAM-1 produced an initial decrease in c.f.u. at $24 \mathrm{~h}$ followed by a mean peak of $5 \cdot 6 \times 10^{3}$ c.f.u. $48 \mathrm{~h}$ after infection, as reported by Lacasse et al. (1990). C. albicans parental strain SC5314 was less virulent than LAM-1 in the oral model, producing a peak of $3.0 \times 10^{3}$ c.f.u. Virulence of C. albicans transformants was also determined in a murine model of systemic candidiasis. In a first experiment, in which survival of mice was used as end point (Fig. 5), no mice died after intravenous infection with strain S1 which was thus completely avirulent. However, strain SC5314 was significantly $(P<0.007)$ more virulent than S2, N1 and N2, which were not significantly different between themselves $(P>0 \cdot 15)$. A second experiment evaluated kidney and liver burdens of the same strains after intravenous infection. Comparison of organ weights revealed no reproducibly significant differences 1,3 and $6 \mathrm{~d}$ after infection with strains SC5314, S1, S2, N1 and N2 (data not shown). However, comparison of mean $\log$ (c.f.u.) recovered from kidneys on these same days showed a strikingly decreased c.f.u. $\mathrm{g}^{-1}$ for strain $\mathrm{S} 1$ compared to the other four strains, which between themselves were 


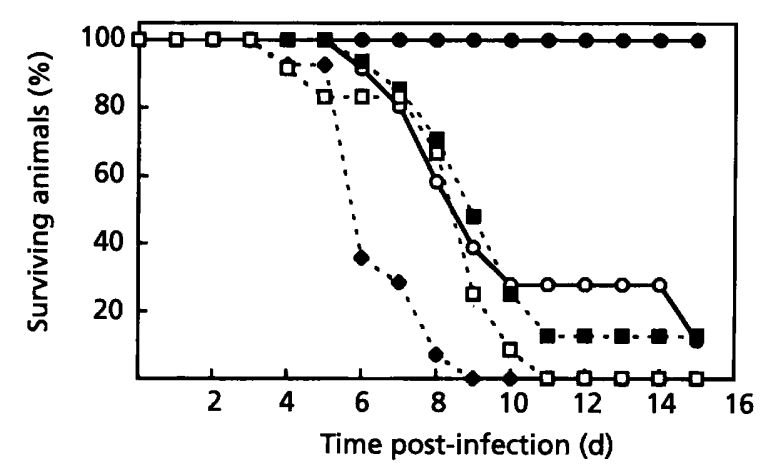

Fig. 5. Survival of CD-1 mice infected intravenously with $5 \times 10^{5}$ cells of $C$. albicans transformants $S 1$ (O, $n=12), S 2(0, n=15)$, N1 $(\square, n=14), N 2(\square, n=12)$ and parental strain SC5314 ( $\bullet$, $n=14)$.

Table 2. Comparative c.f.u. of C. albicans parental strain and transformants recovered from kidneys at various times during disseminated infection of mice

\begin{tabular}{|cccc|}
\hline Day & Strain & No. of animals & $\begin{array}{c}\text { log[c.f.u. (g kidney) } \\
\text { (mean } \pm \text { SD) }\end{array}$ \\
\hline 1 & SC5314 & 7 & $4 \cdot 45 \pm 0 \cdot 75$ \\
& S1 & 7 & $0 \cdot 88 \pm 1 \cdot 39$ \\
& S2 & 7 & $4 \cdot 57 \pm 0 \cdot 27$ \\
& N1 & 4 & $5 \cdot 93 \pm 0 \cdot 07$ \\
& N2 & 5 & $5 \cdot 53 \pm 0.52$ \\
3 & SC5314 & 7 & $5 \cdot 04 \pm 0 \cdot 51$ \\
& S1 & 6 & $0 \cdot 88 \pm 0 \cdot 88$ \\
& S2 & 6 & $5 \cdot 99 \pm 0 \cdot 74$ \\
& N1 & 6 & $5 \cdot 15 \pm 0 \cdot 60$ \\
& N2 & 6 & $5 \cdot 10 \pm 0 \cdot 73$ \\
6 & SC5314 & 7 & $4 \cdot 98 \pm 0 \cdot 59$ \\
& S1 & 6 & 0 \\
& S2 & 4 & $5 \cdot 58 \pm 0 \cdot 15$ \\
& N1 & 5 & $5 \cdot 78 \pm 0 \cdot 28$ \\
& N2 & 6 & $5 \cdot 55 \pm 0.31$ \\
\hline
\end{tabular}

not significantly different (Table 2). Counts in liver were 2 (day 1) and $4 \log$ (c.f.u.) (days 3 and 6) lower than those obtained from the kidneys. However, the relationship between strains remained the same. All strains produced equivalent liver c.f.u. on the three observation days, except for strain $S 1$ which yielded negative cultures (data not shown). Finally, randomly selected colonies of S1, S2, N1 and N2 isolated from the kidneys of mice showed no modification at the SAP2 locus by Southern blotting.

In the previous experiment, one kidney was removed for c.f.u. quantification and the other for histological analysis. Macroscopic examination of the kidneys showed several surface microabcesses 3 and $6 \mathrm{~d}$ after infection for all strains except S1. Interestingly, larger microabcesses were noted for strain S2 after $3 \mathrm{~d}$, compared to the other strains. Histopathological exam- ination demonstrated a mixture of blastoconidia, hyphae and pseudohyphae in the cortex on days 1 and 3 with strains SC5314, N1, N2 and S2. However, no fungi were observed on these same days in kidneys of mice infected with strain S1. Moreover, strain SC5314 produced several cortical abcesses on day 1 , while strains N1, N2 and S2 produced very rare blastoconidia and hyphae. On day 3 , more widespread fungi were seen with strain SC5314 compared to day 1, with extension into the renal medulla; N1, N2 and S2 were very sparse or absent.

\section{Zymolyase and $\mathrm{NaCl}$ resistance}

We observed decreasing viability of $S$. cerevisiae and $C$. albicans transformants expressing or overexpressing Sap2p on CAT medium held for $5 \mathrm{~d}$ at $4{ }^{\circ} \mathrm{C}$, after initial growth at $30^{\circ} \mathrm{C}$ for $48 \mathrm{~h}$. This could have potentially resulted from cell wall damage produced by massive expression and/or secretion of the protease. The same hypothesis could also account for the absence of increased virulence of positive transformant $S 2$ and the decreased virulence of $\mathrm{S} 1$ in vivo, compared to the negative controls. To assess this possibility, two different cell wall resistance assays were done. In both the zymolyase and $\mathrm{NaCl}$ resistance assays, all $S$. cerevisiae negative control and positive transformants demonstrated the same level of sensitivity. However, treatment with zymolyase resulted in a 2 log greater decrease in viability of C. albicans strain S1, compared to SC5314, $\mathrm{S} 2, \mathrm{~N} 1$ and N2. In addition, growth of strain S1 was inhibited by $1.4 \% \mathrm{NaCl}$, compared to $2.2 \%$ for the other four C. albicans strains.

\section{DISCUSSION}

This is the first report describing the construction of stable $S$. cerevisiae and $C$. albicans integrative transformants constitutively secreting Sap2p. We used the $S$. cerevisiae or C. albicans ADH1 promoter (Bertram et al., 1996) on expression plasmids to overexpress $C$. albicans SAP2 in both yeast species. The ADH1 promoter has been used in studies of gene expression in $C$. albicans (Cormack et al., 1997).

On the basis of SAP2 DNA and Sap2p N-terminal sequences, the mRNA is translated as a prepro form, 56 aa larger than the mature protein. The prepropeptide has a signal peptide sequence of 14-21 aa with 1-4 putative signal peptidase cleavage sites and two Lys-Arg sequences, one of which is immediately before the $\mathrm{N}$ terminus of the mature form (Wright et al., 1992; Hube, 1996). Thus, Sap2p was expected to be processed in $S$. cerevisiae because it has been shown that the cleavage of C. tropicalis Sap heterologously expressed in S. cerevisiae is mediated by KEX2-dependent proteolysis (Togni et al., 1996).

The Sap2p signal peptide was functional in S. cerevisiae, allowing the secretion of three active forms of Sap2p. While we cannot rule out the possibility of posttranslational modification, the higher molecular mass 
form $(45 \mathrm{kDa})$ may result from incomplete processing of the proteinase by $S$. cerevisiae. The secretion of a premature form may be due to saturation of the processing system as a consequence of overexpression of the protein. Previous reports have shown that heterologous expression of C. tropicalis SAPT1 (Togni et al., 1996) or C. albicans SAP1 (Smolenski et al., 1997) in S. cerevisiae resulted in secretion of extracellular active enzyme identical to the native form, indicating that no oligosaccharide chains were added. In both cases, expression was under the control of inducible promoter of GAL10 and the hosts were not deficient in intracellular proteinases. Although no extensive studies have been done on the intracellular processing of $C$. albicans Sap2p, Banerjee et al. (1991) and Homma et al. (1992) reported the detection of a $45 \mathrm{kDa}$ intracellular precursor of Sap2p. More recently, it was shown that heterologous expression of SAPT1 of C. tropicalis by $S$. cerevisiae resulted in intracellular forms $4-6 \mathrm{kDa}$ larger than the mature protein (Togni et al., 1996). Further experiments will be required to clarify these issues.

Considering that $S$. cerevisiae is non-pathogenic, the study of the virulence of strains secreting Sap2p is an attractive system to address the role of Sap2p in pathogenicity in the absence of other putative factors. Although episomal expression of Sap1p has been achieved in S. cerevisiae (Smolenski et al., 1997), we have now obtained expression of the major transcription product, Sap2p, from an integrated construct, suitable for studies of virulence in animal models. The constitutive expression of Sap $2 \mathrm{p}$ by $S$. cerevisiae clearly allowed it to hydrolyse and use BSA. This may be important for survival in animal models because it would enable the yeast to use host proteins as nitrogen source.

A model of the regulation of SAP2 expression suggests that C. albicans has a basal level of proteinase expression, which yields peptides that act as inducers of $S A P 2$ expression, via a positive feedback mechanism (Hube et al., 1994). Our data indicate that SAP2 expression was deregulated in those C. albicans strains constitutively producing Sap $2 p$. In the presence of highmolecular-mass protein, the enzyme was secreted precociously, which resulted in earlier induction of the wild-type SAP2 gene and higher amounts of total proteinase activity. These strains constitute a novel approach to explore the role of early and high-level expression of Sap2p during experimental infections. In fact, De Bernardis et al. (1995) have shown that highly vaginopathic strains expressed both SAP1 and SAP2 earlier in experimental vaginitis than moderately or non-vaginopathic strains. Furthermore, it has been hypothesized that Saps may play a role early in systemic dissemination of $C$. albicans to deep organs from mucosal sites (Kimberly et al., 1997). Finally, C. albicans isolates from oral cavities of symptomatic HIV-infected patients secreted significantly higher levels of proteinase than those from asymptomatic or non-infected patients (Ollert et al., 1995; De Bernardis et al., 1996). When tested in experimentally infected animals, the high-level producers had enhanced virulence (De Bernardis et al., 1996). However, whether the increased proteinase activity was due to a particular Sap(s) was not determined.

In pathogenicity studies in vivo, expression of $S A P 2$ as a sole putative virulence factor did not cause an avirulent strain of $S$. cerevisiae to become virulent and constitutive expression of SAP2 did not augment virulence of $C$. albicans in experimental oral or systemic infection in intact mice. The absence of enhanced virulence of $S$. cerevisiae SAP2 transformants is consistent with the long-held view that $C$. albicans possesses a multiplicity of properties, each with a low propensity for enhancing fungal infectivity, none necessarily dominant and all, even in combination, unable to overcome fully intact host defences (Odds, 1988).

Unknown mutations occurring concurrently in all strains would be unlikely to explain the significantly enhanced survival of mice infected with the C. albicans negative control $(\mathrm{N} 1, \mathrm{~N} 2)$ or positive $S A P 2$ transformant (S2), compared to parental strain SC5314. In addition, although $\mathrm{N} 1, \mathrm{~N} 2$ and S2 only contain one copy of URA3, while the wild-type SC5314 has two copies, a gene dosage effect is also unlikely to be responsible for the attenuated virulence of $\mathrm{N} 1, \mathrm{~N} 2$ and S2 since heterozygous $U R A 3$ mutants were as virulent as parental strains in experimentally infected mice (Leberer et al., 1997). Because the negative controls (N1, N2) would have been expected to be as virulent as the wild-type SC5314, mutated SAP2 may have been translated into a truncated protein, resulting in saturation of the secretory pathway.

Although positive C. albicans transformants S1 and S2 were expected to be isogenic, they had different phenotypes. S1 grew much slower in CAT medium compared to all other strains, including the wild-type SC5314. In addition, this strain had higher proteolytic activity in YNB-BSA medium and was avirulent after systemic infection in intact mice. The strikingly decreased virulence of strain S1 and its enhanced cell wall sensitivity compared to the other C. albicans transformants suggest that $S A P 2$ overexpression may have damaged the cell wall, resulting in enhanced killing by polymorphonuclear leukocytes and other intact host defences. Alternatively, an unknown mutation in S1 causing slower growth in CAT medium may also have attenuated virulence. With regard to strain S2, a mutation may have caused a reduced secretion of Sap $2 p$, which in turn caused less damage to the cell wall of C. albicans. Lack of enhancement of virulence of positive C. albicans transformant $\mathrm{S} 2$ compared to negative controls $\mathrm{N} 1$ and $\mathrm{N} 2$ may have resulted from unlikely spurious mutations during transformation, the route of infection, the absence of immunosuppression, or defects in other virulence factors due to saturation of secretory pathways. In this regard, all C. albicans transformants maintained the ability to form pseudohyphae and hyphae in vivo. Although Sap2p is the most abundant transcript of C. albicans yeast cells, increased virulence in vivo may require not only its enhanced expression, but also the early participation of other putative 
virulence factors, including members of the Sap isoenzyme family such as Sap4, 5 and 6 which are produced by the hyphal form of $C$. albicans. Finally, an absence of SAP2 constitutive expression in the animal models was unlikely because $A D H 1$ is a strong constitutive promoter. Furthermore, C. albicans transformants isolated from the kidneys of mice showed no modification at the $S A P 2$ locus. Further studies will be required to determine whether differences in virulence would be observed in a rat vaginal model between the wild-type and strains overexpressing $S A P 2$. Although $S A P 2$ constitutive expression did not augment virulence, this new approach may be a useful paradigm in the study of virulence attributes in Candida and other fungal pathogens.

\section{ACKNOWLEDGEMENTS}

This study was supported by grants from the Medical Research Council of Canada and the Fonds de la Recherche en Santé du Québec. A.R.C. was the recipient of a studentship from the Consejo Nacional de Investigaciones Cientificas y Tecnologicas, Caracas, Venezuela. We are grateful to W. Fonzi for providing C. albicans CAI4, to A. $\mathrm{H}$. Bussey for $S$. cerevisiae cIABYS86 and to K. V. Clemons for S. cerevisiae YJM128, YJM273 and Y55. We also wish to thank A. J. P. Brown for vector YPB-ADH1pL and T. L. Ray for anti-Sap2 mAb. We thank Annie-Claude Miailhe for excellent technical assistance and Marc Fredette, Michel Lamoureux and Yves Lepage for statistical analysis. This work was presented in part at the 97th General Meeting of the American Society for Microbiology, Miami Beach, FL, USA, 4-8 May, 1997.

\section{REFERENCES}

Banerjee, A., Ganesan, K. \& Datta, A. (1991). Induction of secretory acid proteinase in Candida albicans. J Gen Microbiol 137, 2455-2461.

Belhumeur, P., Lee, A., Tam, R., Di Paolo, T., Fortin, N. \& Clark, M. W. (1993). GSP1 and GSP2, genetic suppressors of the prp20-1 mutant in Saccharomyces cerevisiae: GTP-binding proteins involved in the maintenance of nuclear organization. Mol Cell Biol 13, 2152-2161.

Bertram, G., Swoboda, R. K., Gooday, G. W., Gow, N. A. R. \& Brown, A. J. P. (1996). Structure and regulation of the Candida albicans $A D H 1$ gene encoding an immunogenic alcohol dehydrogenase. Yeast 12, 115-127.

Borg, M. \& Ruchel, R. (1988). Expression of extracellular acid proteinase by proteolytic Candida spp. during experimental infection of oral mucosa. Infect Immun 56, 626-631.

Cassone, A., De Bernardis, F., Mondello, F., Ceddia, T. \& Agatensi, L. (1987). Evidence for a correlation between proteinase secretion and vulvovaginal candidosis. J Infect Dis 156, 777-783.

Chakir, J., Côté, L., Coulombe, C. \& Deslauriers, N. (1994). Differential pattern of infection and immune response during experimental oral candidiasis in BALB $/ \mathrm{c}$ and DBA $/ 2\left(\mathrm{H}-2^{d}\right)$ mice. Oral Microbiol Immunol 9, 88-94.

Clemons, K. V., McCusker, J. H., Davis, R. W. \& Stevens, D. A. (1994). Comparative pathogenesis of clinical and nonclinical isolates of Saccharomyces cerevisiae. J Infect Dis 169, 859-867.

Colina, A.-R., Aumont, F., Belhumeur, P. \& de Repentigny, L. (1996a). Development of a method to detect secretory mucinolytic activity from Candida albicans. J Med Vet Mycol 34, 401-406.

Colina, A.-R., Aumont, F., Deslauriers, N., Belhumeur, P. \& de
Repentigny, L. (1996b). Evidence for degradation of gastrointestinal mucin by Candida albicans secretory aspartyl proteinase. Infect Immun 64, 4514-4519.

Cormack, B. P., Bertram, G., Egerton, M., Gow, N. A. R., Falkow, S. \& Brown, A. J. P. (1997). Yeast enhanced-green fluorescent protein $(y E G F P)$ : a reporter of gene expression in Candida albicans. Microbiology 143, 303-311.

Crandall, M. \& Edwards, J. E., Jr (1987). Segregation of proteinasenegative mutants from heterozygous Candida albicans. $J$ Gen Microbiol 133, 2817-2824.

Cutler, J. E. (1991). Putative virulence factors of Candida albicans. Annu Rev Microbiol 45, 187-218.

Dawson-Saunders, B. \& Trapp, R. G. (1990). Basic and Clinical Biostatistics. Norwalk, CT: Appleton \& Large.

De Bernardis, F., Cassone, A., Sturtevant, J. \& Calderone, R. (1995). Expression of Candida albicans SAP1 and SAP2 in experimental vaginitis. Infect Immun 63, 1887-1892.

De Bernardis, F., Chiani, P., Ciccozzi, M., Pellegrini, G., Ceddia, T., D'Offizzi, G., Quinti, I., Sullivan, P. A. \& Cassone, A. (1996). Elevated aspartyl proteinase secretion and experimental pathogenicity of Candida albicans isolates from oral cavities of subjects infected with human immunodeficiency virus. Infect Immun 64, 466-471.

Fonzi, W. A. \& Irwin, M. Y. (1993). Isogenic strain construction and gene mapping in Candida albicans. Genetics 134, 717-728.

Gillum, A. M., Tsay, W. Y. H. \& Kirsch, D. R. (1984). Isolation of the Candida albicans gene for orotidine-5'-phosphate decarboxylase by complementation of the $S$. cerevisiae and E. coli pyrF mutations. Mol Gen Genet 198, 179-185.

Harlow, E. \& Lane, D. (1988). Antibodies: a Laboratory Manual. Cold Spring Harbor, NY: Cold Spring Harbor Laboratory.

Hoffman, C. S. \& Winston, F. (1987). A ten-minute DNA preparation from yeast efficiently releases autonomous plasmids for transformation of Escherichia coli. Gene 57, 267-272.

Homma, M., Kanbe, T., Chibana, H. \& Tanaka, K. (1992). Detection of intracellular forms of secretory aspartic proteinase in Candida albicans. J Gen Microbiol 138, 627-633.

Hube, B. (1996). Candida albicans secreted aspartyl proteinases. Curr Top Med Mycol 7, 55-69.

Hube, B., Monod, M., Schofield, D. A., Brown, A. J. P. \& Gow, N. A. R. (1994). Expression of seven members of the gene family encoding secretory aspartyl proteinases in Candida albicans. Mol Microbiol 14, 87-99.

Hube, B., Sanglard, D., Odds, F. C., Hess, D., Monod, M., Schäfer, W., Brown, A. J. P. \& Gow, N. A. R. (1997). Disruption of each of the secreted aspartyl proteinase genes SAP1, SAP2 and SAP3 of Candida albicans attenuates virulence. Infect Immun 65, 3529-3538.

Kaplan, E. L. \& Meier, P. (1958). Nonparametric estimation from incomplete observations. J Am Stat Assoc 53, 457-481.

Kimberly, F., Bausch, K., Noonan, J., Huguenel, E. \& Tamburini, P. (1997). Role of aspartic proteases in disseminated Candida albicans infection in mice. Infect Immun 65, 551-556.

Kwon-Chung, K. J., Lehman, D., Good, C. \& Magee, P. T. (1985). Genetic evidence for role of extracellular proteinase in virulence of Candida albicans. Infect Immun 49, 571-575.

Lacasse, M., Fortier, C., Trudel, L., Collet, A. J. \& Deslauriers, N. (1990). Experimental oral candidosis in the mouse: microbiological and histological aspects. J Oral Pathol Med 19, 136-141. 
Laemmli, U. K. (1970). Cleavage of structural proteins during the assembly of the head of bacteriophage T4. Nature 227, 680-685.

Leberer, E., Ziegelbauer, K., Schmidt, A., Harcus, D., Dignard, D., Ash, J., Johnson, L. \& Thomas, D. Y. (1997). Virulence and hyphal formation of Candida albicans require the Ste20p-like protein kinase CaCla4p. Curr Biol 7, 539-546.

Luna, G. L. (1992). Histopathologic Methods and Color Atlas of Special Stains and Tissue Artifacts. Downers Grove, IL: Johnson Printers.

Lussier, M., White, A.-M., Sheraton, J. \& 17 other authors (1997). Large scale identification of genes involved in cell surface biosynthesis and architecture in Saccharomyces cerevisiae. Genetics 147, 435-450.

Macdonald, F. \& Odds, F. C. (1983). Virulence for mice of a proteinase-secreting strain of Candida albicans and a proteinasedeficient mutant. J Gen Microbiol 129, 431-438.

Magee, B. B., D'Souza, T. M. \& Magee, P. T. (1987). Strain and species identification by restriction fragment length polymorphisms in the ribosomal DNA repeat of Candida species. J Bacteriol 169, 1639-1643.

Milewski, S., Mignini, F., Covelli, I. \& Borowski, E. (1994). Specific inhibition of acid proteinase secretion in Candida albicans by Lys-Nva-FMDP. J Med Vet Mycol 32, 1-11.

Monod, M., Togni, G., Hube, B. \& Sanglard, D. (1994). Multiplicity of genes encoding secreted aspartic proteases in Candida species. Mol Microbiol 13, 357-368.

Odds, F. C. (1988). Candida and Candidosis, 2nd edn. London: Ballière Tindall.

Ollert, M. W., Wende, C., Görlich, M., McMullan-Vogel, C. G., Borg-von Zepelin, M., Vogel, C.-W. \& Korting, H. C. (1995). Increased expression of Candida albicans secretory proteinase, a putative virulence factor, in isolates from human immunodeficiency virus-positive patients. J Clin Microbiol 33, 2543-2549.

Robinson, J. S., Klionski, D. J., Banta, L. M. \& Emr, S. D. (1988). Protein sorting in Saccharomyces cerevisiae: isolation of mutants defective in the delivery and processing of multiple vacuolar hydrolases. Mol Cell Biol 8, 4936-4948.

Rodrigue, L., Marion, D., Trudel, L., Barthe, C. \& Lavoie, M. C. (1989). Comparison of methods for the evaluation of the oral microbiota of mice. J Microbiol Methods 10, 71-82.

Rose, M. D., Winston, F. \& Hieter, P. (1990). Methods in Yeast Genetics: a Laboratory Course Manual. Cold Spring Harbor, NY: Cold Spring Harbor Laboratory.

Ross, I. K., De Bernardis, F., Emerson, G. W., Cassone, A. \& Sullivan, P. A. (1990). The secreted aspartate proteinase of Candida albicans: physiology of secretion and virulence of a proteinase-deficient mutant. J Gen Microbiol 136, 687-694.

Rothstein, N. (1983). One step gene disruption in yeast. Methods Enzymol 101, 202-211.
Sambrook, J., Fritsch, E. F. \& Maniatis, T. (1989). Molecular Cloning: a Laboratory Manual, 2nd edn. Cold Spring Harbor, NY: Cold Spring Harbor Laboratory.

Sanger, F., Nicklen, S. \& Coulson, A. R. (1977). DNA sequencing with chain-terminating inhibitors. Proc Natl Acad Sci USA 74, 5463-5467.

Sanglard, D., Togni, G., de Viragh, P. A. \& Monod, M. (1992). Disruption of the gene encoding the secreted acid protease (ACP) in the yeast Candida tropicalis. FEMS Microbiol Lett 95, 149-156.

Sanglard, D., Hube, B., Monod, M., Odds, F. C. \& Gow, N. A. R. (1997). A triple deletion of the secreted aspartyl proteinase genes SAP4, SAP5 and SAP6 of Candida albicans causes attenuated virulence. Infect Immun 65, 3539-3546.

Schiestl, R. H., Manivasakam, P., Woods, R. A. \& Gietz, R. D. (1993). Introducing DNA into yeast by transformation. Methods Enzymol 5, 79-85.

Sikorski, R. S. \& Hieter, P. (1989). A system of shuttle vectors and yeast host strains designed for efficient manipulation of DNA in Saccharomyces cerevisiae. Genetics 122, 19-27.

Smolenski, G., Sullivan, P. A., Cutfield, S. M. \& Cutfield, J. F. (1997). Analysis of secreted aspartic proteinases from Candida albicans: purification and characterization of individual Sap1, Sap2 and Sap3 isoenzymes. Microbiology 143, 349-356.

Togni, G., Sanglard, D. \& Monod, M. (1994). Acid proteinase secreted by Candida tropicalis: virulence in mice of a proteinase negative mutant. J Med Met Mycol 32, 257-265.

Togni, G., Sanglard, D., Quadroni, M., Foundling, S. I. \& Monod, M. (1996). Acid proteinase secreted by Candida tropicalis: functional analysis of preproregion cleavages in C. tropicalis and Saccharomyces cerevisiae. Microbiology 142, 493-503.

Towbin, H., Staehelin, T. \& Gordon, J. (1979). Electrophoretic transfer of proteins from polyacrylamide gels to nitrocellulose sheets: procedure and some applications. Proc Natl Acad Sci USA 76, 4350-4354.

Vernet, T., Dignard, D. \& Thomas, D. Y. (1987). A family of yeast expression vectors containing the phage $f 1$ intergenic region. Gene 52, 225-233.

White, T. \& Agabian, N. (1995). Candida albicans secreted aspartyl proteinases: isoenzyme pattern is determined by cell type and levels are determined by environmental factors. J Bacteriol 177 , 5215-5221.

White, T., Kohler, G. A., Miyasaki, S. H. \& Agabian, N. (1995). Expression of virulence factors in Candida albicans. Can J Bot 73 suppl. 1, S1054-S1058.

Wright, R. J., Carne, A., Hieber, A. D., Lamont, I. L., Emerson, G. W. \& Sullivan, P. A. (1992). A second gene for a secreted aspartate proteinase in Candida albicans. J Bacteriol 174, 7848-7853.

Received 26 January 1998; revised 20 April 1998; accepted 24 April 1998. 